\title{
Perfil de suscetibilidade aos antimicrobianos e diversidade das espécies de enterococos isolados de leite cru de búfalas no Sul do Brasil*
}

\section{Antimicrobial susceptibility profile and diversity of enterococci species isolated from raw milk of buffalo in South Brazil}

\author{
Janira Prichula, ${ }^{* *}$ Dejoara de Angelis Zvoboda, ${ }^{* *}$ Rebeca Inhoque Pereira, ${ }^{* *}$ Naiara Aguiar Santestevan, ${ }^{* *}$ \\ Aline Weber Medeiros, ${ }^{* *}$ Amanda de Souza Motta, ${ }^{* *}$ Pedro Alvesd'Azevedo, ${ }^{* * *}$ Altair Roque Giordani, ${ }^{* * * *}$ \\ Ana Paula Guedes Frazzon**
}

\begin{abstract}
Resumo
O leite de búfala é utilizado principalmente para a produção de queijo mussarela. A procura por produtos derivados de leite de búfala tem aumentado no mercado, consideravelmente, nas últimas décadas. Este trabalho teve como objetivo avaliar a diversidade e o perfil de suscetibilidade a antimicrobianos de enterococos isolados de amostras de leite cru de búfalas no Sul do Brasil. Amostras de leite cru refrigerado contendo leite de diversas búfalas cedidas pela Cooperativa Sulriograndense de Bubalinocultores Ind. Com. Ltda, durante junho a agosto de 2012, foram empregadas para isolar as bactérias do gênero Enterococcus. Os microorganismos foram identificados em nível de espécie através de suas características fenotípicas, e seus perfis de suscetibilidade a antimicrobianos foram analisados através do método de disco-difusão em ágar. Oitenta colônias foram selecionadas aleatoriamente das amostras de leite cru e as espécies mais frequentes nestas foram Enterococcus faecalis (63,75\%), seguida de Enterococcus faecium (28,75\%), Enterococcus durans (2,5\%), Enterococcus spp. (3,75\%) e Lactococcus sp. (1,25\%). Os resultados dos testes de sensibilidade revelaram que a maioria dos enterococos era sensível aos antimicrobianos testados. Entretanto, 13,9\% apresentaram perfil de resistência a nitrofurantoína, $12,7 \%$ a tetraciclina, 1,3\% a eritromicina, 1,3\% a norfloxacina, 1,3\% a cloranfenicol e 1,3\% a estreptomicina. Como conclusão, o leite bubalino apresentou uma diversidade de espécie de enterococos semelhante às obtidas em outros alimentos de origem animal. Contudo, a presença de E. faecalis multirresistentes isoladas nesse estudo alerta para a importância da cadeia alimentar na disseminação de resistência aos antimicrobianos de uso clínico fora de ambientes nosocomiais.
\end{abstract}

Palavras-chave: enterococos, leite bubalino, resistência.

\begin{abstract}
Buffalo milk is used mainly for the production of mozzarella cheese. The consumer demand for products manufactured with buffalo milk has increased considerably in recent decades. This study aimed to evaluate the diversity and susceptibility profile of Enterococcus spp. isolated from raw buffalo milk produced in southern region of Brazil. Samples of refrigerated raw milk pooled from several buffaloes were provided by Cooperativa Sulriograndense de Bubalinocultores Ind. Com. Ltda, during the period from June to August 2012, for the evaluation of the Enterococcus genus. Bacterial were identified species by phenotypic characteristics and their susceptibility profiles antibiotic were analyzed using agar disk diffusion method. Eighty colonies were randomly selected from raw milk and in which the most frequent species were Enterococcus faecalis (63.75\%), followed by Enterococcus faecium ( $28.75 \%$ ), Enterococcus durans (2.5\%), Enterococcus spp. (3.75\%) and Lactococcus sp. (1.25\%). The results of antibiotic susceptibility test revealed that the majority of enterococci were susceptible to the antibiotics tested, however $13.9 \%$ were resistant to nitrofurantoin, $12.7 \%$ to tetracycline, $1.3 \%$ to erythromycin, $1.3 \%$ to norfloxacin, $1.3 \%$ to chloramphenicol and $1.3 \%$ to streptomycin. In conclusion, the diversity of enterococci species from buffalo milk were very similar to those obtained in other studies with animal foods, however the presence of $E$. faecalis multiresistant isolated in this study highlights the importance of the food chain in the dissemination of antimicrobial resistance outside of nosocomial environments.
\end{abstract}

Keywords: enterococci, buffalomilk, resistance.

\footnotetext{
*Recebido em 12 de janeiro de 2013 e aceito em 13 de maio de 2013.

**Universidade Federal do Rio Grande de Sul (UFRGS); Instituto de Ciências Básicas da Saúde; Departamento de Microbiologia; Porto Alegre, Rio Grande do Sul, Brasil. Autor para correspondência. E-mail: ana.frazzon@ufrgs.br

***Universidade Federal de Ciências da Saúde de Porto Alegre (UFCSPA); Departamento de Ciências Básicas da Saúde; Porto Alegre, Rio Grande do Sul, Brasil.

${ }^{* * *}$ Cooperativa Sulriograndense de Bubalinocultores Ind. Com. Ltda (COOPERBÚFALO).
} 


\section{Introdução}

Comparativamente com o leite bovino, o leite bubalino apresenta elevado teor de proteínas e minerais, e menor índice de colesterol. A característica sensorial proeminente deste leite é a ausência de pigmentos carotenoides, que lhe confere a coloração branca sem prejudicar a concentração de vitamina A e concomitantemente a qualidade nutricional do leite (Amaral et al., 2005). O leite de búfala é utilizado principalmente para a produção de queijo mussarela. Entretanto, este pode ser empregado na elaboração de outros queijos, como o tipo azul, Minas frescal, requeijão, quark e ricota (Buziet al., 2009).

O leite é um excelente meio de cultura e a qualidade microbiológica da matéria-prima é importante para a fabricação de um derivado de alto padrão. Na Itália, a elaboração da mussarela é feita com leite de búfala não pasteurizado, com o objetivo de não alterar o processo tecnológico, e principalmente garantir as características sensoriais inerentes do produto. No Brasil, muitos laticínios também industrializam leite não pasteurizado (Buziet al., 2009).

Enterococos são bactérias acido laticas que toleraram altas concentrações de sais e variações de $\mathrm{pH}$ e temperatura, podendo sobreviver às temperaturas de pasteurização (Teixeira et al., 2011). O gênero inclui mais de 46 espécies (Euzéby, 2012), sendo Enterococcus faecalis e Enterococcus faecium as mais frequentes em alimentos, plantas, ambiente e trato gastrintestinal de humanos e animais (Pangalloet al., 2004; d'Azevedo et al., 2006; Riboldi et al., 2008, Cassenego et al., 2011). A contaminação dos alimentos ocorre principalmente por meio de contaminações intestinais ou ambientais (Girafa, 2002). A presença de enterococos nos alimentos é preocupante, pois além de ser um indicador de contaminação fecal na produção/ processamento de alimentos, são reservatórios de genes de resistência, possibilitando a disseminação na cadeia alimentar, e propagação de resistência aos antibióticos na população humana. Estudos epidemiológicos salientam que, pela sua natureza oportunista, os enterococos têm emergido como patógenos associados às infecções nosocomiais (Gales et al., 2009; Bender et al., 2009; Lin et al., 2012).

No Brasil, estudos que avaliam a qualidade microbiológica do leite bubalino empregado na produção de queijo mussarela ainda são escassos. Nesse sentido, o objetivo deste estudo foi avaliar a diversidade e o perfil de suscetibilidade aos antimicrobianos de enterococos isolados de amostras de leite cru de búfalas.

\section{Materiais e métodos}

Nesse estudo foram utilizadas quatro amostras de leite cru refrigerado de búfala que foram fornecidas pelo Laticínio Kronhardt pertencente à Cooperativa Sulriograndense de Bubalinocultores Ind. Com. Ltda. Cada amostra continha uma mistura de leites de búfalas de quatro propriedades diferentes que abastecem o Laticínio Kronhardt. Estas propriedades utilizam ordenha mecânica e produzem de 200 a 500 litros por dia, cerca de 10 litros de leite por búfala. As amostras foram cedidas pelo Laticínio Kronhardt, no período de junho a agosto de 2012, e transportadas até o laboratório em caixas isotérmicas contendo gelo reciclável, sendo mantidas sob refrigeração a $\pm 6^{\circ} \mathrm{C}$ até o momento das análises.

Os ensaios de seleção e de isolamento de enterococos foram realizados, conforme Teixeira et al. (2011), em meio seletivo Caldo Azida Dextrose (DAB - Dextrose Azida Broth, Difco
Laboratories, Detroit, MI, USA). Em tubos contendo $9 \mathrm{~mL}$ de $\mathrm{DAB}$ foi inoculado $1 \mathrm{~mL}$ de leite, e então incubados em estufa por 24 horas, a $35^{\circ} \mathrm{C} \pm 2^{\circ} \mathrm{C}$. Após o período de incubação, foram realizadas diluições seriadas até $10^{-5}$, sendo cada uma das 5 diluições plaqueadas em triplicata em ágar Infusão Cérebro Coração (BHI- Brain Heart Infusion, Difco Laboratories, Detroit, MI, USA) acrescido de 6,5\% de $\mathrm{NaCl}$ (Merk Indústrias Químicas S.A., RJ, Brasil). As placas foram incubadas por 24 horas, a $35^{\circ} \mathrm{C} \pm 2^{\circ} \mathrm{C}$. Após o período de incubação, cerca de 25 colônias foram selecionadas aleatoriamente de cada amostra de leite, e semeadas em Agar Bile Esculina (BEA- Bile Esculin Agar, Difco Laboratories, Detroit, MI, USA). Os isolados que apresentavam capacidade de hidrolisar esculina na presença de sais biliares foram semeados em ágar BHI. Posteriormente, verificou-se a produção da enzima catalase utilizando peróxido de hidrogênio $\left(\mathrm{H}_{2} \mathrm{O}_{2}\right)$ a $3 \%(\mathrm{v} / \mathrm{v})$ e a capacidade desses micro-organismos de multiplicar-se a $10^{\circ} \mathrm{C}$ e $45^{\circ} \mathrm{C}$, durante 24 horas.

Os isolados negativos para produção da enzima catalase e com capacidade de crescimento a $10^{\circ} \mathrm{C}$ e $45^{\circ} \mathrm{C}$ foram mantidos em ágar $\mathrm{BHI}$, e submetidos à identificação microscópica preliminar através da verificação das características morfotintoriais em esfregaços corados pelo método de Gram, para visualizar a pureza das colônias. Em seguida, cada isolado foi mantido sob a forma de suspensão em solução contendo $10 \%$ de Skim Milk (Difco Laboratories, Detroit, MI, USA) e glicerol a 10\% (v/v), e armazenados em criotubos a $-20^{\circ} \mathrm{C}$.

Posteriormente, foi realizada a caracterização fenotípica das espécies. Os isolados foram caracterizados presuntivamente em nível de espécie por testes fisiológicos convencionais seguindo as recomendações propostas no Manual of Clinical Microbiology (Teixeira et al., 2011). Esses ensaios incluíram testes para verificar a capacidade dos isolados de utilizar piruvato, hidrolisar arginina, produzir pigmento, tolerar o telurito de potássio, produzir ácidos a partir de diversas fontes de

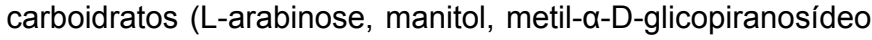
(MGP), D-rafinose, sacarose, D-sorbitol e D-sorbose) e teste de motilidade. Os testes realizados para avaliar a produção de pigmento e a motilidade dos isolados foram realizados apenas nos casos em que estes testes fossem definitivos para identificar alguns isolados. Os ensaios foram realizados com cultivos recentes dos isolados em ágar $\mathrm{BHI}$, obtidos após incubação a $35^{\circ} \mathrm{C} \pm 2{ }^{\circ} \mathrm{C}$, por 24 horas, com os quais foram preparadas suspensões bacterianas em solução salina a 0,85\% esterilizada, com turbidez semelhante à da solução padrão 0,5 de McFarland (aproximadamente 1 a $2 \times 10^{8} \mathrm{UFC} / \mathrm{mL}$ ). Cem microlitros de cada suspensão foram inoculados nos tubos contendo os meios testados, e os tubos foram incubados a $35^{\circ} \mathrm{C} \pm 2^{\circ} \mathrm{C}$ por sete dias. Diariamente, realizaram-se as leituras e interpretações, observando-se alteração de cor dos meios testados devido à mudança de $\mathrm{pH}$. A produção de pigmento foi observada em culturas frescas em ágar BHI, utilizando um swab para observar a presença de coloração amarela das colônias consideradas positivas. O teste para verificação da motilidade foi realizado inoculando-se as amostras bacterianas com o auxílio de agulha bacteriológica em Motility Medium (Difco Laboratories, Detroit, $\mathrm{MI}$, USA), seguido de incubação a $35^{\circ} \mathrm{C} \pm 2^{\circ} \mathrm{C}$ por 24 horas. A observação de turvação indicou resultado positivo. Cepas padrão de E. faecalis ATCC 29212 e E. gallinarum RS-64 foram utilizados como controle para os testes.

$O$ perfil de suscetibilidade aos antimicrobianos foi avaliado apenas para as bactérias pertencentes ao gênero Enterococcus spp., através da utilização do teste de difusão em ágar, conforme 
recomendações do Clinical and Laboratory Standards Institute (CLSI, 2012). Os ensaios foram realizados com cultivos dos isolados em ágar $\mathrm{BHI}$, obtidos após incubação a $35^{\circ} \mathrm{C} \pm 2^{\circ} \mathrm{C}$, por 24 horas, com os quais foram preparadas suspensões bacterianas em solução salina a $0,85 \%$ esterilizada, com turbidez semelhante à da solução padrão 0,5 de McFarland (aproximadamente 1 a $2 \times 10^{8} \mathrm{UFC} / \mathrm{mL}$ ). As suspensões foram semeadas, com o auxílio de swabs esterilizados sobre a superfície seca das placas contendo ágar Mueller-Hinton (Mueller-Hinton Agar, Difco Laboratories, Detroit, MI, USA) para obter-se um crescimento uniforme e confluente. Em seguida, sobre a superfície dos meios inoculados, foram colocados os discos contendo os seguintes antibióticos: nitrofurantoína - NIT $(300 \mu \mathrm{g})$, tetraciclina - TET $(30 \mu \mathrm{g})$, eritromicina - ERI $(15 \mu \mathrm{g})$, norfloxacina - NOR $(10 \mu \mathrm{g})$, cloranfenicol

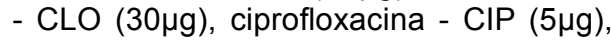
ampicilina - AMP $(10 \mu \mathrm{g})$, vancomicina - VAN

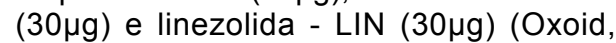
Vasingstoke, UK). Para detecção de níveis elevados de resistência aos aminoglicosídeos (HLR-A) foram utilizados discos de estreptomicina - EST $(300 \mu \mathrm{g})$

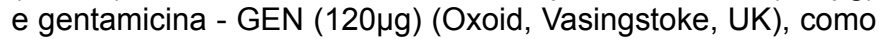
preconiza o CLSI (2012). Após incubação por 24 horas a $35^{\circ} \mathrm{C}$ $\pm 2^{\circ} \mathrm{C}$, foram realizadas leituras e interpretações dos diâmetros dos halos de inibição, segundo as recomendações do CLSI (2012). Para controle de qualidade dos testes de suscetibilidade foram utilizadas as seguintes amostras padrão: Staphylococcus aureus ATCC 25923 e E. faecalis ATCC 29212.

\section{Resultados e discussão}

A partir da seleção e isolamento de enterococos das quatro amostras (A, B, C e D) de leite bubalino, foi isolado um total de 80 colônias. Dezessete colônias foram isoladas da Amostra A, 18 da Amostra B, 24 da Amostra C e 21 da Amostra D. Tais colônias foram capazes de crescer em $\mathrm{DAB}$ e em ágar $\mathrm{BH}$ acrescido de $6,5 \% \mathrm{NaCl}$, hidrolisaram esculina na presença de sais biliares e apresentaram resultado negativo para o teste da catalase. Cresceram em temperatura ótima de $35^{\circ}$.

Além disso, foram capazes de crescer a $10^{\circ} \mathrm{C}$ e $45^{\circ} \mathrm{C}$. Através de coloração pelo método de Gram, foi possível confirmar que todos os isolados testados eram cocos Gram-positivos, isolados, arranjados aos pares ou em cadeias curtas, características típicas apresentadas por enterococos e gêneros relacionados (Murray, 1990; Oplustilet al., 2004; Teixeira et al., 2011).

As 80 colônias isoladas das amostras de leite bubalino foram identificadas em nível de espécie, e a mais frequente foi $E$. faecalis $(63,75 \%)$, seguida de E. faecium $(28,75 \%)$, E. durans (2,5\%), Enterococcus spp. (3,75\%) e Lactococcus sp. (1,25\%). $\mathrm{Na}$ amostra A, 16 colônias foram identificadas como $E$. faecium e uma como $E$. faecalis atípico. $\mathrm{Na}$ amostra $\mathrm{B}, 12$ isolados pertenciam a espécie $E$. faecalis (sendo 5 E. faecalis atípicos), 2 à Enteroccocus durans, 1 à Lactococcus sp. e em 3 isolados não foi possível identificar a espécie. Na amostra $C$ foram identificadas 24 colônias como $E$. faecalis, sendo 7 E. faecalis atípicos. Na amostra D, 14 colônias foram identificadas como $E$. faecalis (onde 3 eram E. faecalis atípicos) e 7 E. faecium (sendo 3 classificados como E. faecium atípicos). A Tabela 1 exibe o perfil fenotípico das bactérias que apresentaram características atípicas.

Tabela 1: Características fenotípicas atípicas das bactérias isoladas de leite ubalino no sul do Brasil

\begin{tabular}{|c|c|c|c|c|c|c|c|c|c|c|}
\hline \multirow[b]{2}{*}{ Espécies } & \multicolumn{10}{|c|}{ Características fenotípicas ${ }^{a}$} \\
\hline & MAN & SOR & ARG & ARA & SBL & RAF & SAC & PIR & MGP & TEL \\
\hline E. faecalis' & + & - & + & $+b$ & + & - & + & + & - & + \\
\hline E. faecalis" & + & - & + & - & + & - & $-\mathrm{b}$ & + & - & $-\mathrm{b}$ \\
\hline E. faecalis ${ }^{\prime \prime \prime}$ & + & - & + & $+^{\mathrm{b}}$ & + & $t_{b}$ & + & + & - & + \\
\hline E. faecium ${ }^{\mathrm{IV}}$ & + & - & + & + & - & + & + & $+^{\mathrm{b}}$ & - & - \\
\hline
\end{tabular}

MAN, manitol; SOR, sorbose; ARG, arginina; ARA, arabinose; SBL, sorbitol; RAF, rafinose; SAC , PIR, piruvato; MGP, metil-a-D-glicopiranosídeo; TEL, telurito de potássio. ${ }^{\mathrm{b}}$ Características atípicas. 'Fenótipo atípico encontrado na amostra $\mathrm{A}$ e $\mathrm{C}$. "'Fenótipo atípico encontrado na amostra $\mathrm{B}$.

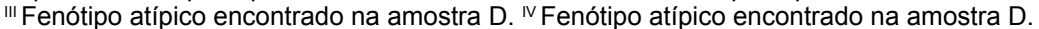

As frequências de espécies de enterococos observadas no presente estudo estão em concordância com os dados da literatura, que registram a maior frequência de $E$. faecalis e $E$. faecium em alimentos de origem animal (Cortés et al., 2006; Tebaldi et al., 2008; Riboldi et al., 2008; Ruzauskas et al., 2009). A predominância de $E$. faecalis em amostras de leite também já foi relatada em outros trabalhos que avaliaram leite bovino pasteurizado (Fracalanzza et al., 2007), leite bovino cru proveniente de tanques de refrigeração (Tebaldi et al., 2008) e amostras de leite bovino e produtos lácteos em Portugal (Lopes et al., 2005). Contudo, não há trabalhos na literatura que relatem a diversidade de enterococos em leite cru de búfala.

A presença de um isolado pertencente ao gênero Lactococcus sp. nas amostras de leite de búfala avaliadas nesse estudo, pode ser justificada pelo fato dessa bactéria também ser um coco Gram-positivo, catalase negativa e produtora de ácido lático, comumente encontrada em amostras de leite (Viani e Lázaro, 2003; Riboldi et al., 2008).

A caracterização fenotípica dos enterococos isolados neste trabalho evidenciou uma alta variabilidade fisiológica de $E$. faecalis e de $E$. faecium. As atipias fisiológicas detectadas neste estudo podem ser justificadas, visto que o método empregado para identificar as espécies de enterococos é baseado no perfil fenotípico de enterococos oriundos de amostras clínicas. Entretanto, atipias também já foram encontradas em outros trabalhos que analisaram enterococos de origem clínica (Resende, 2012), bem como em estudos com enterococos presentes em alimentos de origem animal. Este fato pode ser explicado, pelo menos em parte, pela adaptação metabólica ao substrato oferecido (Fracalanzza, 2007).

Os resultados do perfil de suscetibilidade das espécies de enterococos isoladas de leite bubalino demonstraram uma elevada frequência de isolados suscetíveis aos 11 antimicrobianos testados, conforme verificado na Tabela 2. Esse resultado difere dos dados relatados em estudos no Brasil e também em outros países, que registram uma elevada 
frequência de enterococos resistentes a antimicrobianos isolados de alimentos (Lukásová e Sustácková, 2003; Lopes et al., 2005; Cortés et al., 2006; Fracalanzza et al., 2007; Riboldi et al., 2008). Esta alta frequência de cepas de enterococos sensíveis pode ser explicada pela rusticidade inerente do gado bubalino, o qual apresenta uma maior resistência às doenças infecciosas (Amaral e Escrivão, 2005), não necessitando, assim, da utilização abusiva de antimicrobianos, reduzindo dessa forma a pressão seletiva de bactérias resistentes.

É importante ressaltar que dos 79 enterococos testados, $13,9 \%$ (11/79) apresentaram resistência à nitrofurantoína, 12,7\% (10/79) à tetraciclina, 1,3\% (1/79) ao cloranfenicol, 1,3\% (1/79) à estreptomicina, 1,3\% (1/79) à norfloxacina e 1,3\% (1/79) à eritromicina. Quarenta e sete $(59,5 \%)$ isolados apresentaram resistência intermediária à norfloxacina, $58,2 \%(46 / 79)$ à ciprofloxacina, $51,9 \%(41 / 79)$ à eritromicina e $6,3 \%(5 / 79)$ à nitrofurantoína.

Tabela 2: Perfil de suscetibilidade das espécies de Enterococcus spp. isoladas de leite bubalino no sul do Brasil

\begin{tabular}{|c|c|c|c|c|c|c|c|c|c|}
\hline \multirow{3}{*}{$\begin{array}{c}\text { Ag. } \\
\text { Antimic. }^{a}\end{array}$} & \multicolumn{9}{|c|}{ Percentual de enterococos resistentes } \\
\hline & \multicolumn{3}{|c|}{$\begin{array}{l}\text { E. faecalis } \\
\qquad(n=51)\end{array}$} & \multicolumn{3}{|c|}{$\begin{array}{l}\text { E. faecium } \\
\qquad(\mathrm{n}=23)\end{array}$} & \multicolumn{3}{|c|}{$\begin{array}{l}\text { Outros Enterococcus spp. } \\
\qquad(\mathrm{n}=5)\end{array}$} \\
\hline & $\mathbf{S}$ & $\mathbf{I}$ & $\mathbf{R}$ & $\mathbf{S}$ & $\mathbf{I}$ & $\mathbf{R}$ & $\mathbf{S}$ & $\mathbf{I}$ & $\mathbf{R}$ \\
\hline NIT & 88,2 & - & 11,8 & 78,3 & 21,7 & - & - & - & 100 \\
\hline TET & 80,4 & - & 19,6 & 100 & - & - & 100 & - & - \\
\hline ERI & 45,1 & 52,9 & 2 & 34,8 & 65,2 & - & 100 & - & - \\
\hline NOR & 17,6 & 80,4 & 2 & 73,9 & 26,1 & - & 100 & - & - \\
\hline EST & 98 & - & 2 & 100 & - & - & 100 & - & - \\
\hline CLO & 98 & - & 2 & 100 & - & - & 100 & - & - \\
\hline CIP & 27,5 & 72,5 & - & 60,9 & 39,1 & - & 100 & - & - \\
\hline AMP & 100 & - & - & 100 & - & - & 100 & - & - \\
\hline VAN & 100 & - & - & 100 & - & - & 100 & - & - \\
\hline GEN & 100 & - & - & 100 & - & - & 100 & - & - \\
\hline LIN & 100 & - & - & 100 & - & - & 100 & - & - \\
\hline
\end{tabular}

${ }^{a}$ Agentes Antimicrobianos: NIT, nitrofurantoína; TET, tetraciclina; ERI, eritromicina; NOR, norfloxacina; EST, estreptomicina; CLO, cloranfenicol; CIP, ciprofloxacina; AMP, ampicilina; VAN, vancomicina; GEN, gentamicina; LIN, linezolida.

Enterococos resistentes a antimicrobianos isolados de alimentos foram relatados em estudos no Brasil e em outros países (Lukásová e Sustácková, 2003; Lopes et al., 2005; Cortés et al., 2006; Fracalanzza et al., 2007; Riboldi et al., 2008). Espécies de enterococos resistentes à nitrofurantoína, um antibiótico utilizado para tratamento de infecções no trato geniturinário, também foram identificadas em enterococos isolados de queijos e repolho em Porto Alegre (Riboldi et al., 2008).

Este estudo revelou que $12,7 \%$ das bactérias isoladas do leite de búfala apresentaram fenótipo de resistentes à tetraciclina. Dados da literatura relatam a existência de cepas de enterococos resistentes à tetraciclina em alimentos (Fracalanzza et al.,
2007; Ruzauskas, et al., 2009, Frazzon et al., 2010) e na clínica médica (Bender et al., 2009). Elevados percentuais de resistência intermediária à eritromicina $(51,9 \%)$ foram detectados entre os isolados de leite bubalino. Estes resultados estão de acordo com os dados descritos por Fracalanzza et al. (2007), que também observaram percentuais expressivos $(58,2 \%)$ de resistência intermediária de enterococos isolados de leite bovino pasteurizado e carne de frango. Contrário aos achados de resistência intermediária à norfloxacina $(59,5 \%)$ e à ciprofloxacina $(58,2 \%)$ observadas neste estudo, Fracalanzza et al. (2007), reportaram, apenas $3,2 \%$ e $13,8 \%$ dos enterococos, de resistência intermediária aos respectivos antimicrobianos.

No presente estudo foi observado um grande número de enterococos com perfil de resistência intermediária à norfloxacina e ciprofloxacina (58,2\% e $79 \%$ ). Estas drogas pertencem ao grupo das fluoroquinolonas e quinolonas respectivamente, que são antimicrobianos sintéticos de amplo espectro, ativas contra bactérias Gram-negativas, Gram-positivas e micoplasmas de importância na medicina veterinária, empregados para o tratamento de diversas doenças infecciosas, como em casos de inflamação da glândula mamária (mastite). A literatura não relata testes de sensibilidade aos antimicrobianos em enterococos isolados de amostras de leites bubalinos no Brasil e no mundo e ainda os poucos dados encontrados, referemse ao isolamento/identificação de bactérias resistentes aos antibióticos, de amostras de leite mastítico em rebanhos bubalinos. Cunha et al., 2006, relataram que a penicilina e a tetraciclina foram as drogas que apresentaram a menor eficácia in vitro contra bactérias Gram-positivas e Gram-negativas isoladas de mastite clínica e subclínica de búfalas. Vianni e Lázaro (2003), identificaram Lactococcus garvieae e Enterococcus gallinarum, isoladas do leite de fêmeas com mastite subclínica, apresentando um perfil de resistência contra penicilina/ampicilina $(95,24 \%)$ e tetracilina $(100 \%)$, respectivamente. $\mathrm{O}$ uso indiscriminado de antibióticos em dosagens inadequadas, principalmente em casos de mastite, favorece o desenvolvimento de cepas patogênicas multirresistentes, cuja ocorrência na glândula mamária vem sendo observada frequentemente em casos de mastite subclínica bubalínica (Vianni e Lázaro 2003).

A análise dos perfis de resistência, de acordo com as espécies de enterococos encontradas no leite bubalino, demonstrou percentuais menos elevados de resistência para $E$. durans. Dois isolados de $E$. durans e três de Enterococcus spp. apresentaram resistência à nitrofurantoína e suscetibilidade aos demais antimicrobianos testados. A resistência à nitrofurantoína também já foi encontrada em estudos com $E$. durans isolados de leite de cabra (Cortés et al., 2006). Constatou-se também que $65,2 \%$ (15/23) dos E. faecium isolados do leite bubalino 
apresentaram resistência intermediária à eritromicina, 39,1\% $(9 / 23)$ à ciprofloxacina, $26,1 \%(6 / 23)$ à norfloxacina e $21,7 \%$ $(5 / 23)$ à nitrofurantoína.

Em relação aos $E$. faecalis, 11,8\% (6/51) apresentaram resistência à nitrofurantoína, 19,6\% (10/51) à tetraciclina e 2\% (1/51) foram resistentes à eritromicina, norfloxacina, estreptomicina e cloranfenicol. É importante salientar que 3 (5,9\%) dos E. faecalis apresentaram resistência à tetraciclina e resistência intermediária a pelo menos dois antibióticos de classes diferentes, e um dos isolados de $E$. faecalis foi resistente a 5 classes diferentes de antimicrobianos testadas: a tetraciclina, eritromicina, norfloxacina, estreptomicina e cloranfenicol. Este dado é preocupante, uma vez que bactérias multirresistentes podem representar reservatórios de genes de resistência, sendo capazes de realizar trocas de material genético com as células da microbiota do trato gastrintestinal do hospedeiro humano, e assim, se disseminar na cadeia alimentar. Isso demonstra que os alimentos desempenham um papel importante na propagação de cepas resistentes a antibióticos de uso clínico para a população humana (Giraffa, 2002; Ruzauskas, et al., 2009).

\section{Conclusões}

O leite bubalino apresentou uma diversidade de espécie de enterococos semelhantes às obtidas em outros estudos com alimentos de origem animal, principalmente com o leite bovino. Apesar dos elevados percentuais de enterococos suscetíveis aos antibióticos testados, a presença de cepas multirresistentes em amostras de leite cru representa um problema de saúde pública, uma vez que este gênero é relativamente resistente ao calor, podendo sobreviver às temperaturas de pasteurização do leite, sendo assim uma fonte de disseminação e persistência de enterococos resistentes através da cadeia alimentar.

\section{Agradecimentos}

Os autores agradecem ao Conselho Nacional de Desenvolvimento Científico e Tecnológico, e à Coordenação de Aperfeiçoamento de Pessoal de Nível Superior, pelo suporte financeiro.

\section{Referências}

AMARAL, F.R.; CARVALHO, L.B.; SILVA, N.; BRITO, J.R.F. Qualidade do leite de búfalas: composição. Revista Brasileira de Reprodução Animal, v. 29, n. 2, p. 106-110, 2005.

AMARAL, F.R.; ESCRIVÃO, S.C. Aspectos Relacionados à búfala leiteira. Revista Brasileira de Reprodução Animal, v. 29, n. 2, p. 111-117, 2005.

BENDER, E.A.; FREITAS, A.L.P.; REITER, K.C.; LUTZ, L.; BARTH, A.L. Identification, antimicrobial resistance and genotypic characterization of Enterococcus spp. isolated in Porto Alegre, Brasil. Brazilian Journal of Microbiology, v. 40, p. 693-700, 2009.

BUZI, K. A.; PINTO, J. P. A. N.; RAMOS, P. R. R.; BIONDI, G. F. Análise microbiológica e caracterização do queijo mussarela elaborado a partir de leite de búfala. Ciência e Tecnologia de Alimentos, v. 29, n. 1, p. 7-11. 2009.

CASSENEGO, A.P.V.; d' AZEVEDO, P.A.; RIBEIRO, A.M.L.; FRAZZON, J.; VAN DER SAND, S.T.; FRAZZON, A.P.G. Species distribution and antimicrobial susceptibility of enterococci isolated from broilers infected experimentally with Eimeria spp. and fed with diets containing different supplements. Brazilian Journal of Microbiology, v. 42, n. 2, p. 480-488, 2011.

Clinical and Laboratory Standards Institute (CLSI). Performance standards for antimicrobial susceptibility testing: 22st informational supplement M100-S22. Wayne, PA: CLSI; 2012.

CORTÉS, C.; DE LA FUENTE, R.; CONTRERAS, A.; SÁNCHEZ, A.; CORRALES, J.C.; RUIZ-SANTA-QUITERIA, J.A.; ORDEN, J.A. Occurrence and preliminar study of antimicrobial resistance of enterococci isolated from dairy goats in Spain. Internacional Journal of Food Microbiology, v. 110, p. 100-103, 2006.

CUNHA, A.P.; DA SILVA, L.B.G.; PINHEIRO JÚNIOR, J.W.; DA SILVA, D.R.; OLIVEIRA, A.A. da F.; DA SILVA, K.P.C.; MOTA, R.A. Perfil de sensibilidade antimicrobiana de agentes contagiosos e ambientais isolados de mastite clínica e subclínica de búfalas. Arquivos do Instituto Biológico, v. 73, n. 1, p. 17-21, 2006.

d'AZEVEDO, P.A.; DIAS, C.A.G.; TEIXEIRA, L. M. Genetic diversity and antimicrobial resistance of enterococcal isolates from southern region of Brazil. Revista do Instituto de Medicina Tropical de São Paulo, v. 48, p. 11-16, 2006.
EUZÉBY, J.P. List of Prokaryotic names with Standing in Nomenclature - Genus Enterococcus. Disponível em: http://www. bacterio.cict.fr/e/enterococcus.html. Acesso em: 17 nov. 2012.

FRACALANZZA, S.A.P. Identificação, resistência a antimicrobianos e caracterização molecular de enterococos isolados de alimentos. 2007. 158 f. Tese (Doutorado) - Instituto Nacional de Controle de Qualidade em Saúde - Fundação Oswaldo Cruz, Rio de Janeiro, 2007.

FRACALANZZA, S.A.P.; SCHEIDEGGER, E.M.D.; SANTOS, P.F. dos; LEITE, P.C.; TEIXEIRA, L.M. Antimicrobial resistance profiles of enterococci isolated from poultry meat and pasteurized milk in Rio de Janeiro, Brazil. Memorial Instituto Oswaldo Cruz, v. 102, n. 7, p. 853-859, 2007.

FRAZZON, A.P.G.; GAMA,B.A.; HERMES, V. BIERHALS, C.G.; GUEDES, A.G.;d'AZEVEDO, P.A.; FRAZZON, J. Prevalence of antimicrobial resistance and molecular characterization of tetracycline resistance mediated by tet $(\mathrm{M})$ and tet $(\mathrm{L})$ genes in Enterococcus spp. isolated from food in Southern Brazil. World Journal of Microbiology and Biotechnology, v. 26, p. 365-370, 2010. GALES, A.C.; SADER, H.S.; RIBEIRO, J.; ZOCCOLI, C.; BARTH, A.; PIGNATARI, A.C. Antimicrobial susceptibility of Gram-positive bacteria isolated in brasilian hospitals: participanting in the SENTRY program (2005-2008). The Brazilian Journal of Infectious Diseases, v. 13, n. 2, p. 90-98, 2009.

GIRRAFA, G. Enterococcus from foods. Federation of European Microbiological Societies - Microbiology Reviews, v. 26, p. 163171, 2002.

LIN, Y.T.; HSIEH, K.S.; CHEN, Y.S.; HUANG, I.F.; CHENG, M.F. Infective endocarditis in children without underlying heart disease. Journal of Microbiology, Immunology and Infection, v. XX, p. 1-8, 2012.

LOPES, M.F.S.; RIBEIRO, T.; ABRANTES, M.; MARQUES, J.J.F.; TENREIRO, R.; CRESPO, M.T.B. Antimicrobial resistance profiles of dairy and clinical and type strains of enterococci. Internacional Journal of Food Microbiology,v. 103, p. 191-198, 2005.

LUKÁSOVÁ, J.; SUSTÁCKOVÁ, A. Enterococci and antibiotic resistance.Acta Veterinaria Brno, v. 72, p. 315-323, 2003.

MURRAY, B.E. The life and times of the Enterococcus.Clinical Microbiology Reviews, v. 3, n. 1, p. 46-65, 1990. 
OPLUSTIL, C.P.; ZOCCOLI, C.M.; TOBOUTI, N.R.; SINTO, S.I. Procedimentos Básicos em Microbiologia Clínica. 2. ed. Brasil: São Paulo, 2004, 340 p.

PANGALLO, D., HARICHOVÁ, J., KARELOVÁ, E., DRAHOVSKÁ, H., CHOVANOVA, K., FERIANC, P., TURNA, J.; TIMKO, J. Molecular investigation of enterococci isolated from different environmental sources. Biologia, v. 59, n. 6, p. 829-837, 2004.

RESENDE, M.C.C. Caracterização Fenotípica e Molecular de Enterococcus isolados na cidade de Porto Alegre. 2012. 90 f. Dissertação (Mestrado) - Programa de Pós-graduação em Ciências da Saúde - Universidade Federal de Ciências da Saúde de Porto Alegre, Porto Alegre, 2012.

RIBOLDI, G.P.; FRAZZON, J.; d' AZEVEDO, P.A.; FRAZZON, A.P.G. Antimicrobial resistance profile of Enterococcus spp. isolated from food in southern Brazil. Brazilian Journal of Microbiology, v. 40, p. 125-128, 2009.

RUZAUSKAS, M.; VIRGAILIS, M.; SIUGZDINIENE, R.; SUZIEDELIENE, E.; SEPUTIENEE,V.; DAUGELAVICCIUS, R.; ZIENIUS, D.; SENGAUT, J.; PAVILONIS, A. Antimicrobial resistance of Enterococcus spp. isolated from livestock in Lithuania. Veterinarsky Arhiv, v. 79, n. 5, p. 439-449, 2009.
TEBALDI, V.M.R.; OLIVEIRA, T.L.C.; BOARI, C.A; PICCOLI, R.H. Isolamento de coliformes, estafilococos e enterococos de leite cru provenientes de tanques de refrigeração por expansão comunitários: identificação, ação lipolítica e proteolítica. Ciência e Tecnologia de Alimentos, v. 28, n. 3, p. 753-760, 2008.

TEIXEIRA L.M.; CARVALHO, M.G.; SHEWMAKER, P.L. \& FACKLAM, R.R. Enterococcus. In: VERSALOVIC, J.; CARROLL, K.C.; FUNKE, G.; JORGENSEN, J.H.; LANDRY, M.L.; WARNOCK, D.W. Manual of Clinical Microbiology 10th ed., Washington, DC: American Society for Microbiology Press, 2011, p 350-364.

VIANNI, M.C.E.; LÁZARO, N.S. Perfil de susceptibilidade a antimicrobianos em amostras de cocos Gram-positivos, catalase negativos, isoladas de mastite subclínica bubalina. Pesquisa Veterinária Brasileira, v. 23, n. 2, p. 47-51, 2003. 